\title{
Immunity raised by recent European subtype 1 PRRSV strains allows better replication of East European subtype 3 PRRSV strain Lena than that raised by an older strain
}

\author{
Ivan Trus ${ }^{1 *}$, Ilias S. Frydas ${ }^{1}$, Vishwanatha R. A. P. Reddy ${ }^{1}$, Caroline Bonckaert ${ }^{1}$, Yewei Li ${ }^{1}$, Lise K. Kvisgaard²,
} Lars E. Larsen ${ }^{2}$ and Hans J. Nauwynck ${ }^{1}$

\begin{abstract}
Stable spatial distribution of porcine reproductive and respiratory syndrome (PRRSV)-1 subtypes in Europe is accompanied by a strong population immunity induced by local PRRSV strains. In the present study, it was examined if the immunity induced by three West European subtype 1 PRRSV strains (2007 isolate 07V063 and 2013 isolates $13 \mathrm{~V} 091$ and 13V117) offers protection against the highly virulent East European subtype 3 PRRSV strain Lena. The number of fever days was greater $(p<0.05)$ in the control group ( $7.6 \pm 1.7$ days) compared to the immune groups (07V063-immune: $4.0 \pm 1.2$ days, 13V091-immune: $4.6 \pm 1.1$ days, 13V117-immune: $4.0 \pm 2.9$ days). In all groups, protection was characterized by reduction $(p<0.05$ ) of AUC values of nasal shedding (control: 14.6, 07V063-immune: 3.4, 13V091-immune: 8.9, 13V117-immune: 8.0) and viremia (control: 28.1, 07V063-immune: 5.4, 13V091-immune: 9.0, 13V117-immune: 8.3). Reduction of respiratory disease, nasal shedding (mean AUC and mean peak values) and viremia (mean AUC and mean peak values) was more pronounced in 07V063-immune $(p<0.05)$ than in 13V091immune and 13V117-immune animals. Inoculation with subtype 1 PRRSV strains caused priming of the Lena-specific virus neutralization antibody response. Upon challenge with Lena, we observed a very strong serological booster effect for neutralizing antibodies against strains used for the first inoculation. Our results indicate that inoculation with subtype 1 PRRSV strains can partially protect against antigenically divergent subtype 3 strains. The lower protection level elicited by recently isolated subtype 1 PRRSV strains may impair the outcome of the spatial expansion of subtype 3 strains from East Europe to West Europe.
\end{abstract}

\section{Introduction}

Porcine reproductive and respiratory syndrome virus (PRRSV) is prevalent in most swine farms worldwide, and is a major cause of economic losses and animal suffering. Current genetic analysis of a number of European genotype PRRSV-1 strains reveals the existence of four different subtypes [1]. In Europe, a geographical demarcation exists between areas of low (Western and Central Europe) and high (Eastern Europe) PRRSV-1 diversity

\footnotetext{
*Correspondence: ivan.trus@ugent.be

${ }^{1}$ Laboratory of Virology, Faculty of Veterinary Medicine, Ghent University, Merelbeke, Belgium

Full list of author information is available at the end of the article
}

[1]. New Belgian PRRSV-1 variants still belong to subtype 1 , but genetic changes have led to an increase in virulence and pathogenicity [2]. This drift has resulted in further economic losses in the swine industry in 2013 and 2014. Stability of the spatial distribution of different PRRSV subtypes in Europe allows us to continue using the term "East European subtypes" for subtypes 2, 3 and 4. However, there is a potential risk that East European subtypes, which are genetically and antigenically distinct from Pan-European subtype 1 viruses and are even more virulent/pathogenic, could emerge in Western and Central Europe, leading to a real catastrophe $[1,3,4]$. The role of existing herd immunity in keeping subtypes 2,3 and 4 out of Western and Central Europe is of great interest. 
Active immunization is currently the only widely available and approved way to control PRRS-related problems in swine herds worldwide. The existence of different PRRSV subtypes requires vaccines that induce a strong cross-protective immune response. In a recent publication, it was demonstrated that the PRRSV-1 subtype 1 vaccine strain modified live virus (MLV)-DV (GenBank: KJ127878), which is closely related to the old prototype virus LV (98.4\% identity of full genome sequences) (GenBank: M96262), was able to give partial clinical and virologic protection against PRRSV-1 subtype 3 strain Lena [3]. With the appearance of genetically more distant PRRSV strains, the question raised as to what degree these strains induce a protective immunity against PRRSV Lena.

In the present study, the level of protection against European PRRSV subtype 3 strain Lena was examined in animals immunized with one old and two recent subtype 1 PRRSV-1 strains.

\section{Materials and methods}

Animals, experimental design and inoculation viruses

Twenty conventional pigs were obtained from a PRRSnegative farm. All animals were housed in separate stables in a biosafety level 2 (BSL2) facility at the Faculty of Veterinary Medicine, Ghent University, Belgium. No relevant pathogens (PRRSV, SIV, PCV2) were detected in the animals.

Pigs were randomly divided into four groups with five animals per group (07V063, 13V091, 13V117, and a mock-inoculated control group). Two consecutive inoculations were performed in this experiment. Pigs were 11 weeks old at the first inoculation and 18 weeks old at the second inoculation. Inoculations were performed intranasally using a $2 \times 10^{5}$ tissue culture infectious dose (TCID) with a $50 \%$ end point $\left(\right.$ TCID $_{50}$ ) PRRSV. The 07V063 group was inoculated with the third passage of 07V063 strain: the 13V091 group with the third passage of 13V091 strain; and the 13V117 group with the second passage of 13V117 strain; all propagated in porcine alveolar macrophages (PAMs). These subtype 1 PRRSV-1 strains were isolated from different Belgian farms in 2007 (07V063) and 2013 (13V091 and 13V117). Subtype 1 PRRSV 07V063 strain is a mildly pathogenic Belgian strain $[2,4]$. Newly isolated strains originated from farms with animals experiencing endemic respiratory disorders in nursery pigs (13V091 and 13V117) [2]. The control group was inoculated intranasally using $1 \mathrm{~mL}$ phosphatebuffered saline (PBS) per nostril.

Seven weeks after the first inoculation all four groups were inoculated with the PRRSV Lena strain (forth passage propagated in PAMs). Lena is a highly pathogenic East European subtype 3 PRRSV strain isolated from aborted fetuses on a Belarusian farm [3-5]. Individual sterile syringes and plastic cannulas (Jorgenson Labs J12) were used for intranasal virus inoculation.

The sequences of 07V063, 13V091, 13V117 and Lena were downloaded from GenBank (GenBank: GU737264, KT159248, KT159249, JF802085). Alignment and phylogenetic analysis were performed using the Mobyle@Pasteur web bioinformatics framework [6].

\section{Clinical and pathological examinations}

Clinical monitoring was performed on a daily basis from 3 days before challenge until 21 days post-challenge (dpc). Local parameters included respiratory disorders, discoloration of ears, presence or absence of periocular edema, and diarrhea. Systemic parameters included rectal temperature (with the threshold for fever set at $39.5^{\circ} \mathrm{C}$ ) and liveliness. Local parameters and liveliness were expressed as a score at the animal level, whereas diarrhea was evaluated on a group level. The respiratory scoring system was adapted from Karniychuk et al. [4].

After euthanasia at $35 \mathrm{dpc}$, lungs were collected and a numerical score was given based on the observed macroscopic lung lesions. This score was used to estimate the percentage of lungs affected by pneumonia, as previously described [7].

\section{Virus titration}

Nasal secretions were collected at $0,3,5,7,10,14,21$, 28 and $35 \mathrm{dpc}$ using sterile swabs (COPAN 160C, Copan Italia $\mathrm{SpA}$ ). One swab was used per nostril and per pig, and the two swabs were pooled. One $\mathrm{mL}$ of transport medium was added and after vortexing $\left(1 \mathrm{~h}, 4^{\circ} \mathrm{C}\right)$ the supernatant was collected and stored at $-70{ }^{\circ} \mathrm{C}$ for virus titration. Transport medium was based on buffer solution [1× Dulbecco's PBS (DPBS) with $0.9 \mathrm{mM} \mathrm{CaCl}_{2}, 0.5 \mathrm{mM}$ $\mathrm{MgCl}_{2} \times 6 \mathrm{H}_{2} \mathrm{O}$ and $20 \mathrm{mg} / \mathrm{L}$ phenol red] supplemented with $10 \%$ fetal calf serum (FCS) and a mixture of antibiotics $(1000 \mathrm{IU} / \mathrm{mL}$ penicillin, $1 \mathrm{mg} / \mathrm{mL}$ streptomycin and $0.5 \mathrm{mg} / \mathrm{mL}$ gentamycin). Virus titers in the supernatant and serum were determined by titration on PAMs collected from PRRSV negative pigs, as previously described [8]. The monoclonal antibody 13E2 against the nucleocapsid protein of PRRSV was used to detect PRRSVinfected cells. Plain swabs were weighed before and after swabbing, and viral titers were calculated per $100 \mathrm{mg}$ of secrete [4]. Fifty percent endpoint titers were calculated by the Reed-Muench method [9].

\section{Serological examinations}

Blood was collected at $0,3,5,7,10,14,21,28$ and 35 $\mathrm{dpc}$ by puncture of the vena cava cranialis, and serum samples were prepared after centrifugation $(10 \mathrm{~min}$, $\left.1800 \mathrm{~g}, 4{ }^{\circ} \mathrm{C}\right)$. PRRSV-specific antibodies were detected 
by the immunoperoxidase monolayer assay (IPMA), and PRRSV-specific neutralizing antibodies were titrated using the virus neutralization (VN) test on MARC-145 cells, as previously described [10]. Serum samples were heat-inactivated for $30 \mathrm{~min}$ at $56{ }^{\circ} \mathrm{C}$ prior to testing. The PRRSV strain Lena was used as an antigen in IPMA and $\mathrm{VN}$ assays. Additional $\mathrm{VN}$ assays were performed with PRRSV strains 07V063, 13V091 and 13V117 to detect neutralizing antibodies in animals immunized with the respective strains.

\section{Statistical analysis}

Serological titers (IPMA and VN), as well as viral loads, were $\log$-transformed prior to analysis. Samples testing negative were assigned a value corresponding to half the minimum detectable titer. Gross pathology scores and area under the curve (AUC) were analyzed using the non-parametric Kruskal-Wallis test with Dunn's post-test. Statistical analysis of continuous data was performed using repeated-measures two-way analysis of variance (rANOVA), with Bonferroni's post-test. Results were considered to be significantly different at $p<0.05$. All data were expressed as the mean value \pm standard deviation (SD).

\section{Results}

\section{Genetic relationship between subtype 1 strains and East} European subtype 3 PRRSV strains

A low level of identity was calculated between the pairwise distances of full genome sequences of the PRRSV subtype 3 Lena strain and the PRRSV subtype 1 strains 07V063 (79.9\%), 13V091 (79.6\%) and 13V117 (79.8\%) (Figure 1).

Phylogenetic analysis of full genome sequences revealed a high identity level (99.9\%) between the 07V063 and 13V117 strains (Figure 1). Non-synonymous

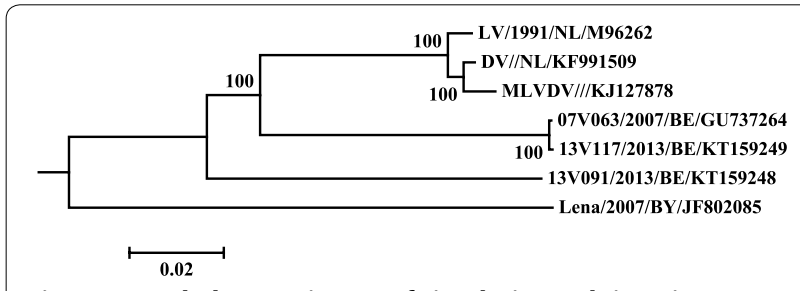

Figure 1 A phylogenetic tree of circulating Belgian virus

isolates. Molecular phylogenetic analysis of full genome nucleotide sequences was constructed using the Neighbor-Joining method. Phylogenetic relationships were estimated using the Clustal Omega method. Prototype subtype 1 (LV) and subtype 3 (Lena) PRRSV-1 strains, vaccine parental (DV) and attenuated (MLV-DV) PRRSV-1 strains were added to phylogenetic tree. The optimal tree is drawn to scale. Numbers indicate bootstrap values of 100 replicates. Strain nomenclature is as follows: name or diagnostic number/year of isolation/country of origin/GenBank accesion number. nucleotide substitutions resulted in eight different amino acids located in non-structural protein (nsp) 1 (V140M), nsp2 (H786L, G1300S), nsp7 (S2192N), nsp9 (M72I, M266V), GP3 (N253H) and GP4 (Q72P). Two were located in putative antigenic epitopes [11-13]. Q72P was found in the B cell ES12 epitope and neutralizing antigenic region (NAR) GP4.16 [11, 12]. N253H was found in NAR GP3.62 [12].

\section{Clinical signs and pathological examination}

Body temperature and respiratory disease scores are represented in Figure 2. Fever was observed after challenge with PRRSV Lena in animals from 2 to $11 \mathrm{dpc}$ in the control group, from 3 to $9 \mathrm{dpc}$ in the 07V063-immune group, from 3 to $7 \mathrm{dpc}$ in the 13V091-immune group, and from 3 to $8 \mathrm{dpc}$ in the 13V117-immune group. The number of fever days was higher $(p<0.05)$ in the control group (7.6 \pm 1.7 days) compared to the immune groups (07V063-immune: $4.0 \pm 1.2$ days, 13V091-immune: $4.6 \pm 1.1$ days, 13 V117-immune: $4.0 \pm 2.9$ days). Significantly higher mean temperature $(p<0.01)$ was observed in the 13V091-immune group $\left(40.9 \pm 0.5^{\circ} \mathrm{C}\right)$ compared to the control group $\left(39.5 \pm 0.7^{\circ} \mathrm{C}\right)$ at $3 \mathrm{dpc}$. Mean AUC values had no significant differences between the groups (control group: $4.5 \pm 2.9,07 \mathrm{~V} 063$-immune group: $2.4 \pm 0.9$, 13V091-immune group: $3.6 \pm 1.2$, 13V117-immune group: $2.5 \pm 2.6$ ). In all groups, animals showed no diarrhea, nasal discharge, coughing, or ear discoloration.

Animals from the 07V063-immune group showed significantly lower mean clinical scores for respiratory disease than control animals at $3 \mathrm{dpc}(p<0.05)$. One animal from the 13V091-immune group, and three animals from the control and 13V117-immune groups had clinical scores $>1$. No animals from the 07V063-immune group had such a level of respiratory disease.

The extent of macroscopic pneumonia was not significantly different $(p>0.05)$. The lowest values were observed in the 07V063-immune group (control group: $1.6 \pm 1.8 \%$, 07V063-immune group: $0.3 \pm 0.4 \%$, 13V091immune group: $1.0 \pm 2.2 \%$, $13 \mathrm{~V} 117$-immune group: $1.5 \pm 1.4 \%)$.

\section{Virological analysis}

Nasal shedding (Figure 3) was observed from $3 \mathrm{dpc}$ in the control, 13V091-immune and 13V117-immune animals, and from $5 \mathrm{dpc}$ in animals from the 07V063immune group. At 5-7 dpc, shedding was found in all animals except for one pig from the 13V117-immune group. A peak mean virus titer was reached at 3 (control group: $10^{4.3} \mathrm{TCID}_{50} / 100 \mathrm{mg}$ ) or $5 \mathrm{dpc}$ (07V063-immune group: $10^{2.4} \mathrm{TCID}_{50} / 100 \mathrm{mg}$, 13V091-immune group: $10^{4.6} \mathrm{TCID}_{50} / 100 \mathrm{mg}$, 13V117-immune group: $10^{4.1}$ 


\section{Body temperature}

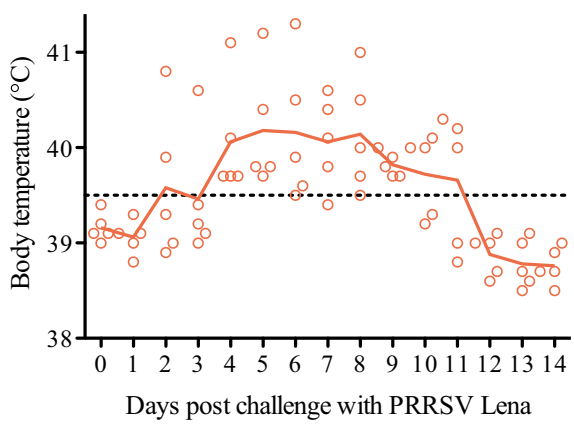

07V063immune

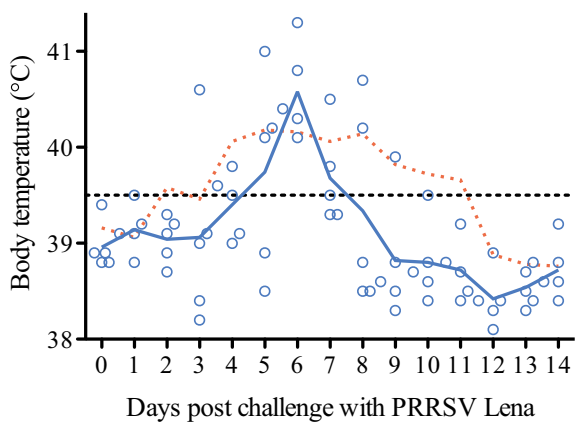

13V091immune

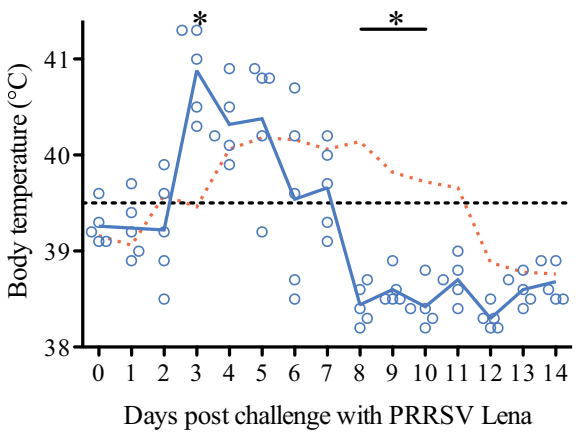

13V117immune

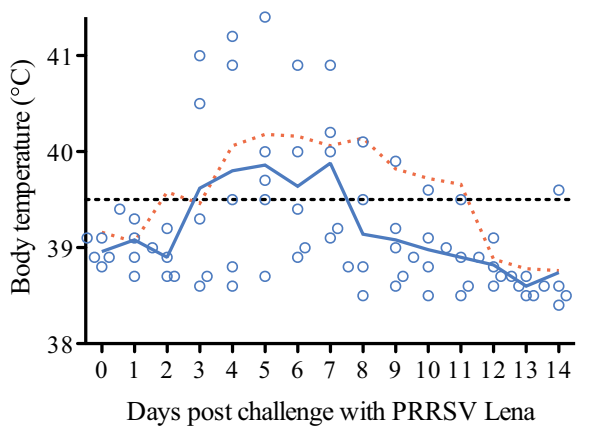

Respiratory disease
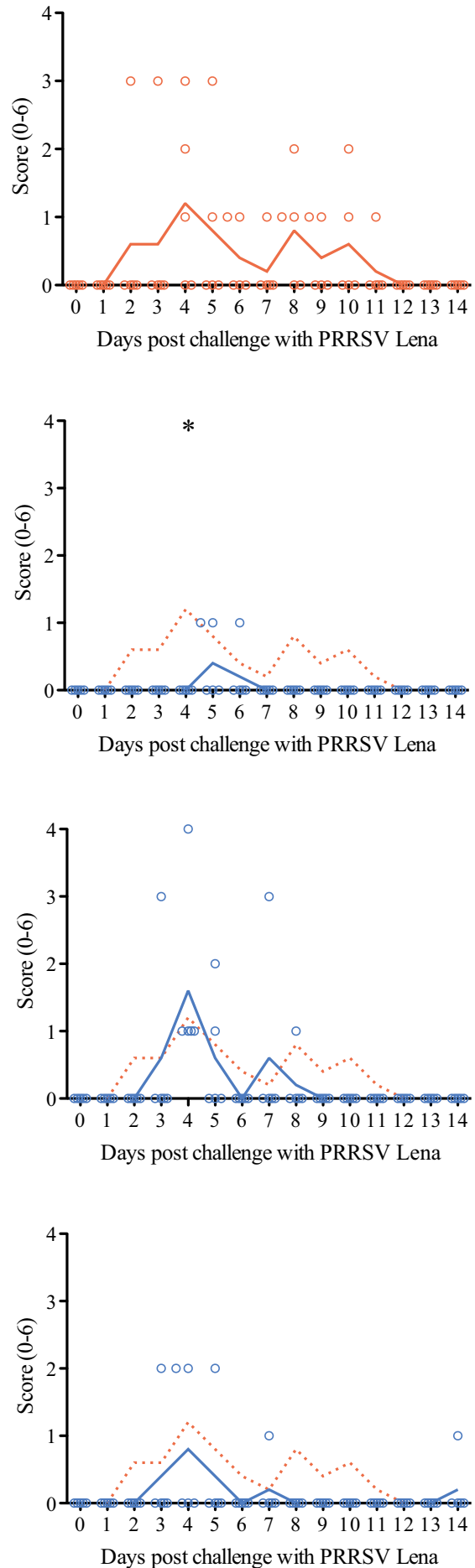

Figure 2 Body temperature and respiratory disease scoring after challenge. Lines represent the mean value in each group. Dotted line gives the mean values of the control group. Temperatures of $\geq 39.5^{\circ} \mathrm{C}$ were considered as fever (dashed line). Respiratory disease scores ranged from 0 to 6: $0=$ normal; $1=$ mild dyspnea and/or tachypnea when stressed; $2=$ mild dyspnea and/or tachypnea at rest; $3=$ moderate dyspnea and/or tachypnea when stressed; 4 = moderate dyspnea and/or tachypnea at rest; 5 = severe dyspnea and/or tachypnea when stressed; $6=$ severe dyspnea and/or tachypnea at rest. Asterisk represents statistically discernible differences from the control $(p<0.05)$. 
Nasal shedding
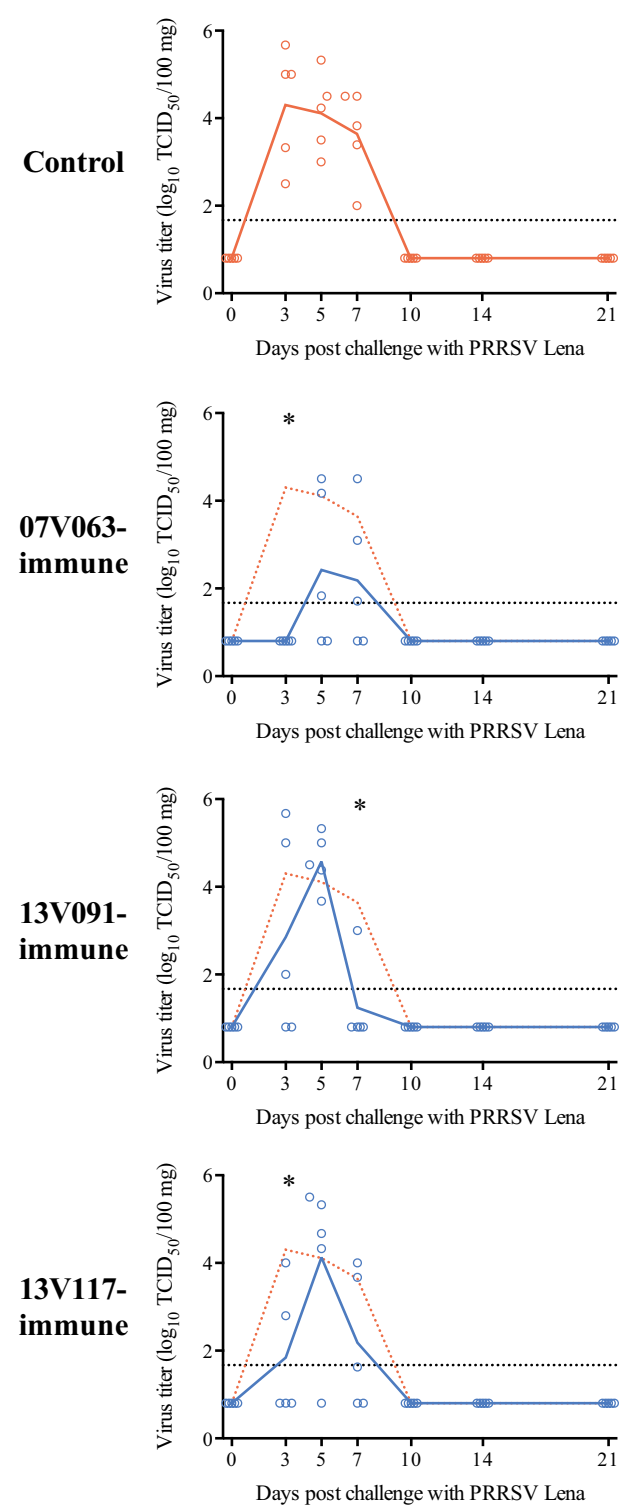

Viremia
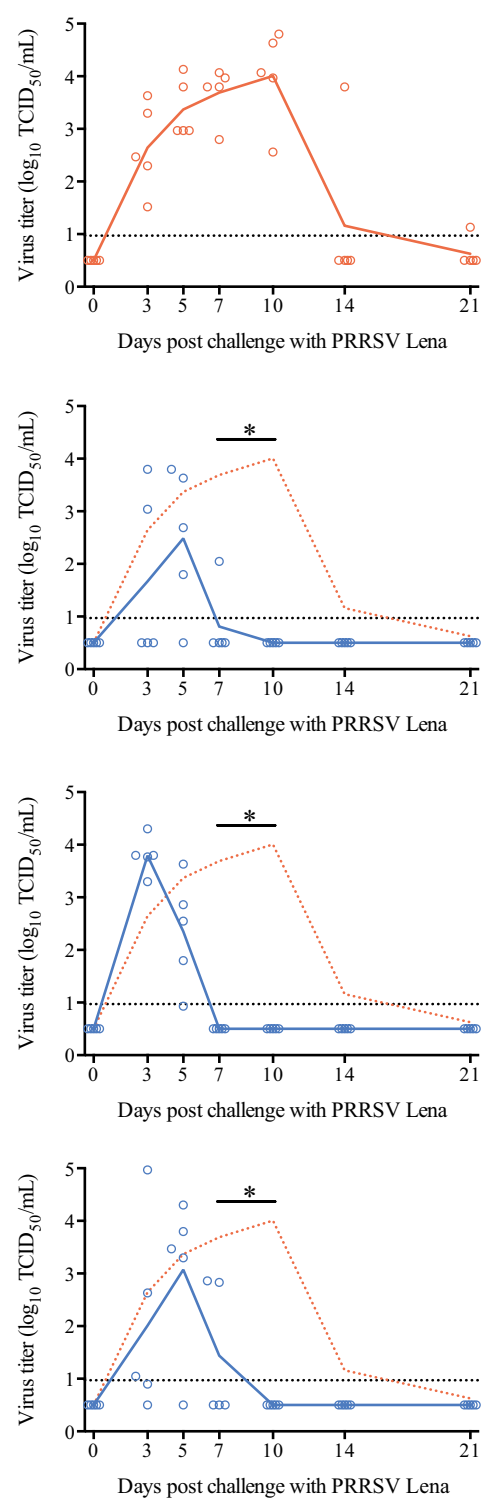

Clustering of viremia (AUC) and nasal shedding (AUC)

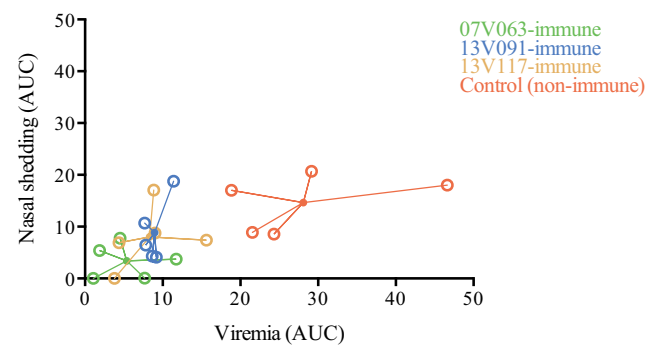

Figure 3 PRRSV titers in nasal secretions and sera after challenge and clustering of individual AUCs. Lines represent the mean titer in each group. Dotted line gives the mean values for the control group. Dashed line gives the detection limit for virus titration. Asterisk represents statistically discernible differences from the control $(p<0.05)$. 
TCID $\left._{50} / 100 \mathrm{mg}\right)$. Significantly lower mean virus titers were detected at $3 \mathrm{dpc}$ in 07V063-immune and 13V117immune animals, and at $7 \mathrm{dpc}$ in 13V091-immune animals compared to control pigs. Nasal shedding stopped in all animals by $10 \mathrm{dpc}$. The mean AUC was significantly lower $(p<0.05)$ in the 07V063-immune group than in the control group (control group: $14.6 \pm 5.6$, 07V063immune group: $3.4 \pm 3.4$, 13V091-immune group: $8.9 \pm 6.1$, 13V117-immune group: $8.0 \pm 6.1$ ) (Figure 3).

Viremia was detected from $3 \mathrm{dpc}$ in all animals, except for one 13V117-immune animal and in three animals from the 07V063-immune group (Figure 3). Virus was isolated from all animals at 5-7 dpc. A peak mean virus titer was observed at $10 \mathrm{dpc}$ (control group: $10^{4.0} \mathrm{TCID}_{50} / \mathrm{mL}$ ), $5 \mathrm{dpc}$ (07V063-immune group: $10^{2.5}$ $\mathrm{TCID}_{50} / \mathrm{mL}$, 13V117-immune group: $10^{3.1} \mathrm{TCID}_{50} / \mathrm{mL}$ ) or $3 \mathrm{dpc}$ (13V091-immune group: $\left.10^{3.8} \mathrm{TCID}_{50} / \mathrm{mL}\right)$. Significantly higher virus titers were found in the control group compared to all immune groups at 7 and $10 \mathrm{dpc}$ $(p<0.001)$. No virus was isolated from serum samples starting from $28 \mathrm{dpc}$ (control group), $10 \mathrm{dpc}$ (07V063immune and 13V117-immune groups) or 7 dpc (13V091immune group). The mean AUC was significantly higher $(p<0.05)$ in control animals than in 07V063-immune and 13V117-immune groups (control group: $28.1 \pm 11.0$, 07V063-immune group: $5.4 \pm 4.4$, 13V091-immune group: $9.0 \pm 1.5,13 \mathrm{~V} 117$-immune group: $8.3 \pm 4.8$ ). The relationship between the AUCs of nasal shedding and viremia was found to be significant (Spearman's rho $=0.62, p=0.0038$ ) (Figure 3 ).

\section{Serology}

Prior to challenge, serum samples animals in the control group were negative according to the IPMA (Figure 4). Animals from all immune groups had high titers of virusspecific antibodies $\left(2^{7.3}-2^{15.3}\right)$. The antibody titers in immune and control animals were not different from 10 dpc $(p>0.05)$.

VN antibodies against subtype 1 PRRSV strains (07V063, 13V091 and 13V117) were detected at $0 \mathrm{dpc}$ in five animals from the 07V063-immune group, four animals from the 13V091-immune group and five out of five animals from the 13V117-immune group (Figure 4). Individual $\mathrm{VN}$ antibody titers increased fourfold at $7 \mathrm{dpc}$ in 07V063, 13V091 and 13V117-immune groups.

Prior to challenge, VN antibodies against the subtype 3 PRRSV Lena strain were detected only in one animal from the 13V091-immune group (Figure 4). Upon challenge, two out of five animals in the control group had a detectable level of $\mathrm{VN}$ antibodies at 21 and $28 \mathrm{dpc}$. In immune groups, the majority of animals (>50\%) demonstrated a VN antibody response against Lena strain during the experiment: 07V063-immune group: five animals,

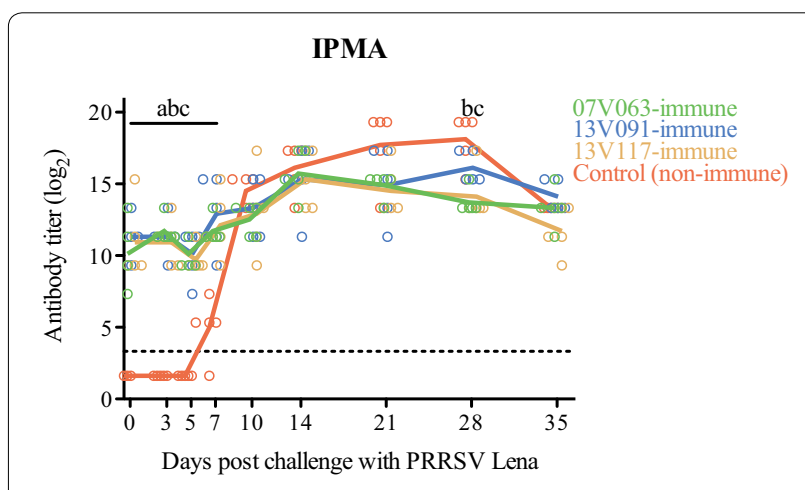

VN titers (against subtype 1 strains)

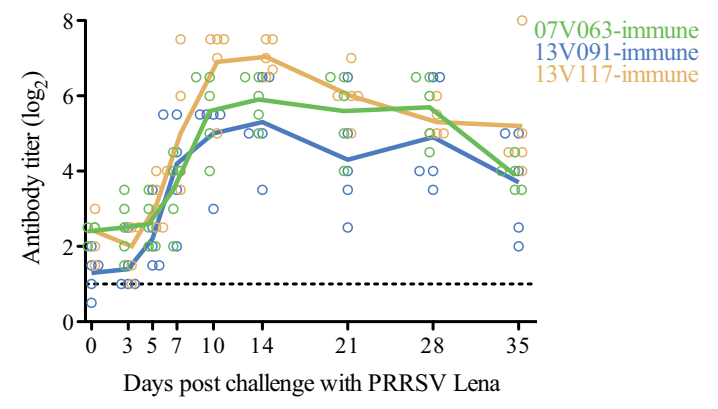

VN titers (against subtype 3 Lena strain)

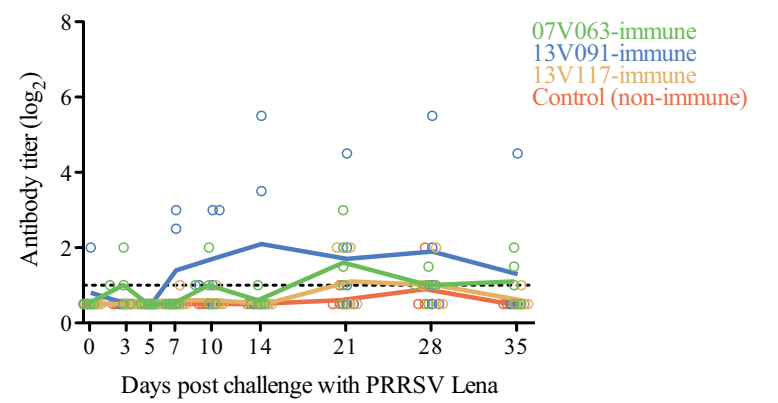

Figure 4 PRRSV-specific IPMA and VN antibody titers after challenge. The PRRSV Lena strain was used as an antigen in IPMA assay. VN antibody titers were tested against subtype 1 (07V063, 13V091 and 13V117, respectively) and against subtype 3 PRRSV (Lena) strains. Lines represent the mean titer in each group. Dashed line gives detection limit for the test. Letters (a: 13V091-immune group, b: 13V117-immune group, c: 07V063-immune group) represent statistically significant differences from the control $(p<0.05)$.

13V091-immune group: four animals, 13V117-immune group: three animals).

\section{Discussion}

A high rate of genetic and antigenic variability among PRRSV isolates hampers effective prevention and control of the disease by the use of commercial vaccines. Rising diversity of PRRSV makes it impossible to have a reference isolate representing the whole country or a part of 
Europe. In the present study, we examined the protection of the immunity induced by an old and two new West European subtype 1 PRRSV strains effective against a heterologous East European subtype 3 virus challenge. The three subtype 1 isolates that were used in this study (07V063, 13V091 and 13V117) differed strongly genetically from subtype 3 PRRSV strain Lena. Detailed reports on their genetic backgrounds and the pathogenicity of these subtype 1 and 3 PRRSV strains have been previously published [1-5].

As an outcome of this study, protection in all immunized groups was characterized by reduction of fever length $(p<0.05)$, nasal shedding (mean AUC) and viremia (mean AUC). Reduction of respiratory disease, nasal shedding (mean AUC and mean peak values) and viremia (mean AUC and mean peak values) was more pronounced $(p<0.05)$ in animals inoculated with the older, low pathogenic strain (07V063-immune group). Subtype 1 PRRSV 13V091 and 13V117 strains were isolated in 2013, 6 years later than the 07V063 strain. A lower level of cross protection caused by immunization with the most recent strains, illustrates a negative impact of rising evolutional diversity of contemporary PRRSV strains on the formation of heterosubtypic immunological protection in swine herds, and on the preservation of spatial distribution of PRRSV subtypes in Europe.

Cellular immunity and neutralizing antibodies may be involved in clearance of the virus after PRRSV infection. The latter may correlate with the protective immunity against PRRSV [14]. In this study, inoculation of animals with subtype 1 PRRSV strains caused priming of Lenaspecific VN antibodies. We observed a faster VN antibody response upon challenge with PRRSV Lena in the 07V063-immune group (50\% of the animals formed a VN antibody response at $3 \mathrm{dpc}$ ) compared to the other groups (control group: >35 dpc, 13V091-immune group: $10 \mathrm{dpc}$, 13V117-immune group: $21 \mathrm{dpc}$ ). Moreover, only in the 07V063-immune group was a VN antibody response against PRRSV Lena detected in all animals. Faster VN antibody response in animals from the 07V063-immune group coincides with better clinical and virologic protection. Thus, VN antibody response upon challenge may be considered as an important factor in the development of immunologic protection.

Upon challenge with subtype 3 PRRSV Lena, a booster effect was observed against strains used for the first inoculation. Analysis of putative antigen recognition domains revealed conservation for two of them [11-13]. One neutralizing antigenic region (GP2.30) was conserved in all of the strains used in this study (EHSGQAAWKQVV) [12]. One epitope (HGAGNMGVDGSVWDF) from nsp9 with $\mathrm{T}$-cell antigen reactivity was conserved in Lena, 07V063 and 13V117 strains [13]. Although subtype 3 strain Lena is genetically and antigenically quite different from the subtype 1 PRRSV strains (07V063, 13V091 and 13V117), neutralizing epitopes (e.g., GP2.30) may stay conserved and provide the $\mathrm{VN}$ antibody booster reaction.

Possible exacerbation of clinical effects $[15,16]$, intensive interstitial pneumonia [17] and increased viremia [18] has already been reported in animals immunized with DNA, sub-unit, and MLV vaccines against PRRS, respectively. We observed that immunization with the 07V063 strain, compared to the 13V091 and 13V117 strains, provided substantially better cross-protection against the subtype 3 PRRSV Lena strain. In contrast, animals from the 13V091-immune group demonstrated temporal exacerbation of PRRS disease manifested by higher body temperature $(p<0.001)$ and viremia $(p<0.05)$ at $3 \mathrm{dpc}$. Compared to the 07V063-immune group, 13V091-immune animals had a higher body temperature $(p<0.001)$, respiratory disease and viremia $(p<0.01)$ at $3 \mathrm{dpc}$, and higher virus titers in nasal secretions at 3-5 dpc $(p<0.05)$. Taken together, these data suggest that the immune response against PRRSV might be a double-edged sword [19]. On the one hand, it provides protection against genetically close and distant strains, but on the other hand it may activate early replication after contact by an antibody-dependent enhancement of infectivity (ADEI) process [18]. In the current study, genetic relatedness may not be a useful guide to accurately predict the level of cross-protection in pigs. Further research is needed to assess the mechanism involved in the immunopathogenesis of PRRS.

In the present study, immunization of animals with subtype 1 PRRSV strains resulted in partial protection, and significantly reduced viral replication and clinical signs upon inoculation with a heterologous East European subtype 3 PRRSV Lena strain. These results could be compared with the outcome of our recent study on the efficacy of an attenuated vaccine (Porcilis ${ }^{\circledR}$ PRRS, MSD Animal Health) based on the subtype 1 PRRSV DV strain (GenBank: KJ127878, KF991509) [3]. PRRSVspecific IPMA antibody titers after one shot vaccination were similar to those observed at $0 \mathrm{dpc}$ in the current study. Animals were challenged with subtype 3 PRRSV Lena strain in the same way as in this study. Protection in the vaccination study was characterized by a similar reduction of clinical disease, i.e., reduced fever length $(p<0.05)$ and respiratory distress level $(p<0.05)$. Virologic parameters showed protection with reduced viremia $(p<0.05)$ and shorter duration of nasal shedding $(p<0.05)$, and lower AUCs for viremia $(p<0.05)$ and nasal shedding $(p<0.05)$ as in the present study. Similarly to the current experiment, the majority $(>50 \%)$ of vaccinated animals had detectable levels of $\mathrm{VN}$ antibodies after challenge ( $14 \mathrm{dpc}$ for the vaccination at 4 weeks, 28 
$\mathrm{dpc}$ for the vaccination at 7 weeks). Overall, we can conclude that parenteral administration of the widely used MLV vaccine against PRRS and immunization with subtype 1 strains is able to induce a comparable protection level against the subtype 3 PRRSV strain Lena.

Immunization of pigs should be used to prevent the transmission of recently isolated highly pathogenic PRRSV strains. However, currently available commercial vaccines based on old type 1 PRRSV strains provide only partial protection against genetically different strains from the same type $[3,20]$. Therefore, the use of live-resident virus inoculation (LVI) may be proposed. This procedure, which has been evaluated as PRRS management tool $[19,21]$, consists of exposure of naïve gilts prior to introducing them into the breeding herd. Although the immune response to virulent PRRSV is usually stronger than that to attenuated virus [19], the LVI approach can increase the risk of further dissemination of field PRRSV strains. In addition, higher production losses in breeding herds were shown when the LVI method was used compared to farms using MLV vaccines [19]. Therefore, the use of MLV vaccines should be preferred for PRRS control and elimination programs.

The restricted flow of marketable pigs from Eastern to Western Europe is keeping the risk of transboundary spread of highly pathogenic subtype 3 PRRSV strains to a minimum. In our study we investigated the role of immunity on the outcome of such spread. Although the immunity elicited by all three subtype 1 PRRSV strains provides a partial protection against antigenically divergent subtype 3 strains, a lower protection level was induced by the two most recently isolated subtype 1 PRRSV strains (2013) compared to that induced by the older strain (2007). The higher replication of a subtype 3 PRRSV strain in pigs immune after infection with more recent strains might result in an easier spatial expansion of subtype 3 strains nowadays than in the past.

\section{Abbreviations}

ADEl: antibody-dependent enhancement of infectivity; AUC: area under the curve; BSL2: biosafety level 2; DPBS: Dubecco's phosphate buffered saline; dpc: days post-challenge; FCS: fetal calf serum; IPMA: immunoperoxidase monolayer assay; LVI: live-resident virus inoculation; MLV: modified live virus; nsp: non-structural protein; PAM: porcine alveolar macrophage; PBS: phosphate buffered saline; PRRS: porcine reproductive and respiratory syndrome; PRRSV: porcine reproductive and respiratory syndrome virus; rANOVA: repeatedmeasures two-way analysis of variance; SD: standard deviation; TCID: tissue culture infectious dose; VN: virus neutralization.
\end{abstract}

\section{Competing interests}

The authors declare that they have no competing interests.

\section{Author details}

${ }^{1}$ Laboratory of Virology, Faculty of Veterinary Medicine, Ghent University, Merelbeke, Belgium. ${ }^{2}$ National Veterinary Institute, Technical University of Denmark, Frederiksberg C, Denmark.

\section{Authors' contributions}

HJN, IT, ISF, CB, VRAPR and YL conceived and designed the experiment. IT, ISF, CB, VRAPR and YL performed the experiment. LKK and LEL undertook full genome sequencing and genetic analyses. IT performed analysis and prepared the manuscript. All authors read and approved the final manuscript.

\section{Acknowledgements}

The authors wish to acknowledge Chantal Vanmaercke and Carine Boone for their excellent technical assistance, and Zeger Van den Abeele and Loes Geypen for helping with animal housing and handling. The local Ethical and Animal Welfare Committee of the Faculty of Veterinary Medicine of Ghent University approved the animal experiment (EC 2010/090). This work was funded by the European Union Seventh Framework Programme (FP7/2007-2013) under grant agreement no 245141.

Received: 22 June 2015 Accepted: 10 December 2015

Published online: 08 January 2016

\section{References}

1. StadejekT, Stankevicius A, Murtaugh MP, Oleksiewicz MB (2013) Molecular evolution of PRRSV in Europe: current state of play. Vet Microbiol 165:21-28

2. Frydas IS, Trus I, Kvisgaard LK, Bonckaert C, Reddy VR, Li Y, Larsen LE, Nauwynck HJ (2015) Different clinical, virological, serological and tissue tropism outcomes of two new and one old Belgian type 1 subtype 1 porcine reproductive and respiratory virus (PRRSV) isolates. Vet Res 46:37

3. Trus I, Bonckaert C, van der Meulen K, Nauwynck HJ (2014) Efficacy of an attenuated European subtype 1 porcine reproductive and respiratory syndrome virus (PRRSV) vaccine in pigs upon challenge with the East European subtype 3 PRRSV strain Lena. Vaccine 32:2995-3003

4. Karniychuk UU, Geldhof M, Vanhee M, Van Doorsselaere J, Saveleva TA Nauwynck HJ (2010) Pathogenesis and antigenic characterization of a new East European subtype 3 porcine reproductive and respiratory syndrome virus isolate. BMC Vet Res 6:30

5. Weesendorp E, Stockhofe-Zurwieden N, Popma-De Graaf DJ, Fijten H, Rebel JM (2013) Phenotypic modulation and cytokine profiles of antigen presenting cells by European subtype 1 and 3 porcine reproductive and respiratory syndrome virus strains in vitro and in vivo. Vet Microbiol 167:638-650

6. Néron B, Ménager H, Maufrais C, Joly N, Maupetit J, Letort S, Carrere S, Tuffery P, Letondal C (2009) Mobyle: a new full web bioinformatics framework. Bioinformatics 25:3005-3011

7. Halbur PG, Paul PS, Frey ML, Landgraf J, Eernisse K, Meng XJ, Lum MA, Andrews JJ, Rathje JA (1995) Comparison of the pathogenicity of two US porcine reproductive and respiratory syndrome virus isolates with that of the Lelystad virus. Vet Pathol 32:648-660

8. Delputte PL, Vanderheijden N, Nauwynck HJ, Pensaert MB (2002) Involvement of the matrix protein in attachment of porcine reproductive and respiratory syndrome virus to a heparinlike receptor on porcine alveolar macrophages. J Virol 76:4312-4320

9. Reed $\amalg$, Muench $H$ (1938) A simple method for estimating fifty percent endpoints. Am J Epidemiol 27:493-497

10. Vanhee M, Delputte PL, Delrue I, Geldhof MF, Nauwynck HJ (2009) Development of an experimental inactivated PRRSV vaccine that induces virus-neutralizing antibodies. Vet Res 40:63

11. Oleksiewicz MB, Bøtner A, Toft P, Normann P, Storgaard T (2001) Epitope mapping porcine reproductive and respiratory syndrome virus by phage display: the nsp2 fragment of the replicase polyprotein contains a cluster of B-cell epitopes. J Virol 75:3277-3290

12. Vanhee M, Van Breedam W, Costers S, Geldhof M, Noppe Y, Nauwynck H (2011) Characterization of antigenic regions in the porcine reproductive and respiratory syndrome virus by the use of peptide-specific serum antibodies. Vaccine 29:4794-4804

13. Mokhtar H, Eck M, Morgan SB, Essler SE, Frossard JP, Ruggli N, Graham SP (2014) Proteome-wide screening of the European porcine reproductive and respiratory syndrome virus reveals a broad range of $\mathrm{T}$ cell antigen reactivity. Vaccine 32:6828-6837 
14. Lopez OJ, Osorio FA (2004) Role of neutralizing antibodies in PRRSV protective immunity. Vet Immunol Immunopathol 102:155-163

15. Díaz I, Ganges L, Galindo-Cardiel I, Tarradas J, Alvarez B, Lorca-Oró C, Pujols J, Gimeno M, Darwich L, Domingo M, Dominguez J, Mateu E (2013) Immunization with DNA vaccines containing porcine reproductive and respiratory syndrome virus open reading frames 5, 6, and 7 may be related to the exacerbation of clinical disease after an experimental challenge. Viral Immunol 26:93-101

16. Prieto C, Martínez-Lobo FJ, Díez-Fuertes F, Aguilar-Calvo P, Simarro I, Castro JM (2011) Immunisation of pigs with a major envelope protein sub-unit vaccine against porcine reproductive and respiratory syndrome virus (PRRSV) results in enhanced clinical disease following experimental challenge. Vet J 89:323-329

17. Pirzadeh B, Dea S (1998) Immune response in pigs vaccinated with plasmid DNA encoding ORF5 of porcine reproductive and respiratory syndrome virus. J Gen Virol 79:989-999
18. Yoon KJ, Wu LL, Zimmerman JJ, Hill HT, Platt KB (1996) Antibodydependent enhancement (ADE) of porcine reproductive and respiratory syndrome virus (PRRSV) infection in pigs. Viral Immunol 9:51-63

19. Linhares DC, Cano JP, Torremorell M, Morrison RB (2014) Comparison of time to PRRSv-stability and production losses between two exposure programs to control PRRSv in sow herds. Prev Vet Med 116:111-119

20. Martelli P, Cordioli P, Alborali LG, Gozio S, De Angelis E, Ferrari L, Lombardi G, Borghetti P (2007) Protection and immune response in pigs intradermally vaccinated against porcine reproductive and respiratory syndrome (PRRS) and subsequently exposed to a heterologous European (Italian cluster) field strain. Vaccine 25:3400-3408

21. Batista L, Pijoan C, Torremorell M (2002) Experimental injection of gilts with porcine reproductive and respiratory syndrome virus (PRRSV) during acclimatization. J Swine Health Prod 10:147-152

\section{Submit your next manuscript to BioMed Central and we will help you at every step:}

- We accept pre-submission inquiries

- Our selector tool helps you to find the most relevant journal

- We provide round the clock customer support

- Convenient online submission

- Thorough peer review

- Inclusion in PubMed and all major indexing services

- Maximum visibility for your research

Submit your manuscript at www.biomedcentral.com/submit
(O) Biomed Central 